\title{
REGULATION OF LEAD-BASED AMMUNITION AROUND THE WORLD
}

\author{
DOMINIQUe AVERY AND RICHARD T. WATSON \\ The Peregrine Fund, 5668 West Flying Hawk Lane, Boise, ID 83709, USA. \\ E-mail: rwatson@peregrinefund.org
}

\begin{abstract}
The use of lead shot and bullets has been regulated in many countries around the world. Using published literature, we compiled a summary of the extent, type, reason, and date for establishing ammunition legislation in each country where it exists. We documented 29 countries with regulations on lead ammunition. The types of bans varied widely and ranged from partial, voluntary restrictions of the use of lead shot to a total ban on the use and import of lead ammunition. The most common restriction $(n=14)$ was the ban of lead shot for hunting of waterfowl over wetlands. The reason for the ban of lead ammunition was most often due to concerns over populations of waterfowl or avian scavengers. Many countries created legislation in response to the African-Eurasian Waterfowl Agreement's (AEWA) recommendation for the use of nontoxic shot over wetlands. Other countries, such as Liberia, banned the use of lead ammunition after a military coup. A timeline demonstrates the momentum with which this issue is gaining ground with most of the regulations taking place in the past 15 years and further regulations under discussion in many areas. An accumulating body of evidence shows that a reduction in the use of lead for hunting also benefits wildlife and humans who consume wild game. Received 16 May 2008, accepted 25 July 2008.
\end{abstract}

AVERY, D., AND R. T. WATSON. 2009. Regulation of lead-based ammunition around the world. In R. T. Watson, M. Fuller, M. Pokras, and W. G. Hunt (Eds.). Ingestion of Lead from Spent Ammunition: Implications for Wildlife and Humans. The Peregrine Fund, Boise, Idaho, USA. DOI 10.4080/ilsa.2009.0115

Key words: Ammunition, country, lead, regulation, state, world.

THE USE OF LEAD SHOT AND BULLETS is regulated in many countries around the world. The types of regulation vary widely and range from partial, voluntary restrictions of the use of lead shot to a total ban on the use and import of lead ammunition. Most countries have implemented regulations due to concerns about the health of migratory waterfowl and avian scavengers. Existing regulations are being strengthened and new ones implemented due to accumulating evidence of the adverse health effects in wildlife and humans of lead from spent ammunition.

\section{METHODS}

We used the internet to search for reports and peerreviewed articles on lead ammunition regulation.
We compiled a summary of the extent, type, reason, and date for establishing lead-based ammunition legislation in each country where it exists. Data that could not be verified for accuracy were excluded.

\section{RESULTS}

Our search yielded 29 countries that have implemented voluntary or legislative restrictions on the use of lead ammunition (Table 1). Two counties have banned all forms of lead ammunition. Six countries have a partial ban on the use of lead bullets in addition to full bans on lead shot. Four countries have banned the use of lead shot for all hunting. Fourteen countries and Australian territories have banned the use of lead shot in wetlands or for waterfowl hunting. Two countries have voluntary 
or recommended restrictions in place. Eleven countries and Australian territories have a partial ban on lead shot. Twenty-five states of the United States have implemented regulations on the use of lead shot in addition to the Federal guidelines. Seven countries have implemented increasingly strict regulations on lead ammunition over time.

\section{DISCUSSION}

Many countries created legislation in response to the African-Eurasian Waterfowl Agreement's (AEWA) recommendation for the use of nontoxic shot over wetlands (Beintema 2001). Concern about populations of avian scavengers have prompted bans in several countries, such as Japan, and in the United States lead ammunition has been banned in portions of California used by condors. Liberia, where lead shot was banned due to a military coup, was the only country to ban lead ammunition for reasons other than health of wildlife or humans.

Increasingly strict regulation imposed on the use of lead ammunition is a growing trend internationally. A timeline (Table 2) demonstrates the momentum with which this issue is gaining ground with most of the regulations taking place in the past 15 years and further regulations under discussion in many areas. Scandinavian countries have led the way in a full ban on lead ammunition, with Denmark ban- ning lead in 2000 and Sweden scheduled to implement a full ban in 2008 .

Evidence of lead exposure in Arctic subsistence hunters who continue to use lead shot (Dewailly et al. 2001, Johansen et al. 2003) suggests that the ban on behalf of eagles has benefited humans as well. Countries worldwide are responding to an accumulating body of evidence that shows that the reduction in the use of lead-based ammunition for hunting benefits wildlife and humans who consume wild game.

\section{Literature Cited}

BEINTEMA, N. 2001. Lead poisoning in waterbirds. International Update Report 2000. Wetlands International and UNEP/African-Eurasian Waterbird Agreement Secretariat, Bonn, Germany.

Dewailly, E., P. Ayott, S. Bruneau, G. Lebel, P. LeVAllois, AND J. P. WeBer. 2001. Exposure of the Inuit population of Nunavik (Arctic Quebec) to lead and mercury. Archives Environmental Health 56:350-357.

Johansen, P., G. ASMUnd, AND F. Riget. 2004. High human exposure to lead through consumption of birds hunted with lead shot. Environmental Pollution 127:125-129. 
Table 1. Comparison of types of lead-based ammunition regulation worldwide in 2008.

Asterisk indicates states or other sub-regions of countries.

\begin{tabular}{|c|c|c|c|c|c|c|c|c|}
\hline $\begin{array}{l}\text { Country } \\
\text { or State }\end{array}$ & $\begin{array}{l}\text { Recommended use } \\
\text { of nontoxic shot }\end{array}$ & $\begin{array}{l}\text { Partial ban } \\
\text { on lead shot }\end{array}$ & $\begin{array}{l}\text { Ban on lead } \\
\text { shot in wet- } \\
\text { lands or for } \\
\text { waterfowl }\end{array}$ & $\begin{array}{l}\text { Ban on lead } \\
\text { shot for all } \\
\text { hunting }\end{array}$ & $\begin{array}{l}\text { Partial ban } \\
\text { on lead } \\
\text { ammunition }\end{array}$ & $\begin{array}{l}\text { Ban on all } \\
\text { forms of lead } \\
\text { ammunition }\end{array}$ & $\begin{array}{l}\text { Ban on } \\
\text { hunting }\end{array}$ & $\begin{array}{l}\text { Nontoxic } \\
\text { shot regu- } \\
\text { lations in } \\
\text { addition to } \\
\text { Federal } \\
\end{array}$ \\
\hline Austria & Banned prior to 2002 & & & & & & & \\
\hline \multicolumn{9}{|l|}{ Australia } \\
\hline $\begin{array}{r}{ }^{*} \text { Capital } \\
\text { Territory, AU }\end{array}$ & & & & & & & $\begin{array}{l}\text { Hunting } \\
\text { ban on } \\
\text { native } \\
\text { wildlife }\end{array}$ & \\
\hline $\begin{array}{r}\text { *Western } \\
\text { Australia, AU }\end{array}$ & & & & & & & $\begin{array}{l}\text { Hunting } \\
\text { ban on } \\
\text { duck and } \\
\text { quail }\end{array}$ & \\
\hline $\begin{array}{r}\text { *South Australia, } \\
\text { AU }\end{array}$ & & $\begin{array}{l}\text { Banned during duck } \\
\text { season, } 1998\end{array}$ & & 1993 & & & & \\
\hline $\begin{array}{r}{ }^{*} \text { Northern } \\
\text { Territory, AU }\end{array}$ & & $\begin{array}{l}\text { Banned in hunting } \\
\text { reserves, } 1998\end{array}$ & & & & & & \\
\hline $\begin{array}{r}{ }^{*} \text { Queensland, } \\
\text { AU }\end{array}$ & 2001 & Banned at three sites & & & & & $\begin{array}{l}\text { Hunting } \\
\text { ban on } \\
\text { duck and } \\
\text { quail, } 2005\end{array}$ & \\
\hline${ }^{*}$ Tasmania, AU & & & 2004 & & & & & \\
\hline $\begin{array}{l}\text { *New South } \\
\text { Wales, AU }\end{array}$ & & Ban for duck hunting & & & & & $\begin{array}{l}\text { Hunting } \\
\text { ban on } \\
\text { duck }\end{array}$ & \\
\hline *Victoria, AU & & $\begin{array}{l}\text { Banned for duck } \\
\text { hunting, } 1993\end{array}$ & 1995 & & & & & \\
\hline Denmark & 1985 & & 1993 & 1996 & & $\begin{array}{l}\text { Ban on } \\
\text { the import } \\
\text { of lead } \\
\text { ammunition, } \\
2000\end{array}$ & & \\
\hline Belgium & & $\begin{array}{l}\text { Banned in Ramsar } \\
\text { sites, } 1993\end{array}$ & 1998 & $\begin{array}{l}\text { Ban } \\
\text { considered } \\
\text { for } 2008\end{array}$ & & & & \\
\hline Canada & & & 1997 & $\begin{array}{l}1999 \text { lead } \\
\text { shot banned } \\
\text { for hunting } \\
\text { game birds }\end{array}$ & & & & \\
\hline
\end{tabular}


- AVERy AND WATSON -

\begin{tabular}{|c|c|c|c|c|c|c|c|c|}
\hline $\begin{array}{l}\text { Country } \\
\text { or State }\end{array}$ & $\begin{array}{l}\text { Recommended use } \\
\text { of nontoxic shot }\end{array}$ & $\begin{array}{l}\text { Partial ban } \\
\text { on lead shot }\end{array}$ & $\begin{array}{l}\text { Ban on lead } \\
\text { shot in wet- } \\
\text { lands or for } \\
\text { waterfowl }\end{array}$ & $\begin{array}{l}\text { Ban on lead } \\
\text { shot for all } \\
\text { hunting }\end{array}$ & $\begin{array}{l}\text { Partial ban } \\
\text { on lead } \\
\text { ammunition }\end{array}$ & $\begin{array}{l}\text { Ban on all } \\
\text { forms of lead } \\
\text { ammunition }\end{array}$ & $\begin{array}{l}\text { Ban on } \\
\text { hunting }\end{array}$ & $\begin{array}{l}\text { Nontoxic } \\
\text { shot regu- } \\
\text { lations in } \\
\text { addition to } \\
\text { Federal }\end{array}$ \\
\hline Cyprus & & & 1993 & & & & & \\
\hline Finland & & & 1996 & & & & & \\
\hline France & & & 2006 & & & & & \\
\hline Germany & 1993 & Ban in 10 states & & & & & & \\
\hline Ghana & & & & & & & $\begin{array}{l}\text { Hunting } \\
\text { ban in } \\
\text { wetlands } \\
\text { and } \\
\text { irrigation } \\
\text { sites }\end{array}$ & \\
\hline Hungary & & & 2005 & & & & & \\
\hline India & & & & & & & $\begin{array}{l}\text { All hunting } \\
\text { banned }\end{array}$ & \\
\hline Israel & & & & & & & $\begin{array}{l}\text { Most } \\
\text { wetlands } \\
\text { closed to } \\
\text { hunting- } \\
\text { must use } \\
\text { lead shot }\end{array}$ & \\
\hline Italy & & & $\begin{array}{l}\text { Proposed } \\
\text { date } \\
\text { unknown }\end{array}$ & & & & & \\
\hline Japan & & & & & $\begin{array}{l}\text { Partial ban on } \\
\text { lead ammuni- } \\
\text { tion for deer, } \\
2000\end{array}$ & & & \\
\hline \multicolumn{9}{|l|}{ Kenya } \\
\hline Latvia & & $\begin{array}{l}\text { Banned in wetland } \\
\text { SPA's, } 2000\end{array}$ & & & & & & \\
\hline Liberia & & & $\begin{array}{l}\text { Military coup } \\
\text { banned lead } \\
\text { shot, } 1980\end{array}$ & & & & & \\
\hline Malaysia & & Date unknown & & & & & & \\
\hline Malta & & $\begin{array}{l}\text { Banned in two } \\
\text { wetlands }\end{array}$ & & & & & & \\
\hline
\end{tabular}




\begin{tabular}{|c|c|c|c|c|c|c|c|c|}
\hline $\begin{array}{l}\text { Country } \\
\text { or State }\end{array}$ & $\begin{array}{l}\text { Recommended use } \\
\text { of nontoxic shot }\end{array}$ & $\begin{array}{l}\text { Partial ban } \\
\text { on lead shot }\end{array}$ & $\begin{array}{l}\text { Ban on lead } \\
\text { shot in wet- } \\
\text { lands or for } \\
\text { waterfowl }\end{array}$ & $\begin{array}{l}\text { Ban on lead } \\
\text { shot for all } \\
\text { hunting }\end{array}$ & $\begin{array}{l}\text { Partial ban } \\
\text { on lead } \\
\text { ammunition }\end{array}$ & $\begin{array}{l}\text { Ban on all } \\
\text { forms of lead } \\
\text { ammunition }\end{array}$ & $\begin{array}{l}\text { Ban on } \\
\text { hunting }\end{array}$ & $\begin{array}{l}\text { Nontoxic } \\
\text { shot regu- } \\
\text { lations in } \\
\text { addition to } \\
\text { Federal }\end{array}$ \\
\hline Mauritania & & & & & & $\begin{array}{l}\text { Ban on all } \\
\text { lead for large } \\
\text { game and } \\
\text { sport hunting } \\
1975\end{array}$ & & \\
\hline Netherlands & & & & 1993 & $\begin{array}{l}\text { Banned for } \\
\text { clay pigeon } \\
\text { shooting, } \\
2004\end{array}$ & & & \\
\hline Norway & & & 1991 & 2005 & & & & \\
\hline Poland & Recommended & & & & & & & \\
\hline Portugal & & & $\begin{array}{l}\text { Proposed for } \\
2008\end{array}$ & & & & & \\
\hline Russia & & $\begin{array}{l}\text { Some restrictions for } \\
\text { wetlands }\end{array}$ & & & & & & \\
\hline South Africa & & $\begin{array}{l}\text { Partial ban on lead } \\
\text { shot for waterfowl }\end{array}$ & & & & & & \\
\hline Spain & & $\begin{array}{l}\text { Banned in Ramsar } \\
\text { sites in } 1994\end{array}$ & 2001 & & & & & \\
\hline Sweden & & & 2002 & & $\begin{array}{l}\text { Banned for } \\
\text { clay pigeon } \\
\text { shooting, } \\
2002\end{array}$ & 2008 & & \\
\hline Switzerland & & & 1998 & & & & & \\
\hline \multicolumn{9}{|l|}{ Great Britain } \\
\hline${ }^{\star}$ England & & Voluntary ban in 1995 & 1999 & & & & & \\
\hline *Scotland & & & 2005 & & & & & \\
\hline${ }^{*}$ Wales & & $\begin{array}{l}\text { Banned in SSSI } \\
\text { wetlands } 2002\end{array}$ & & & & & & \\
\hline New Zealand & & $\begin{array}{l}10 \text { or } 12 \text { gauge shot } \\
\text { banned, } 2006\end{array}$ & & & & & & \\
\hline United States & & & 1991 & & & & & \\
\hline $\begin{array}{r}\text { *Tejon Ranch, } \\
\text { CA }\end{array}$ & & & & & & 2008 & & \\
\hline $\begin{array}{r}{ }^{*} \text { Camp Roberts, } \\
\text { CA }\end{array}$ & & & & & & 2007 & & \\
\hline *Alabama & & & & & & & & \\
\hline
\end{tabular}


- AVERy AND WAtson -

\begin{tabular}{|c|c|c|c|c|c|c|c|c|}
\hline $\begin{array}{l}\text { Country } \\
\text { or State }\end{array}$ & $\begin{array}{l}\text { Recommended use } \\
\text { of nontoxic shot }\end{array}$ & $\begin{array}{l}\text { Partial ban } \\
\text { on lead shot }\end{array}$ & $\begin{array}{l}\text { Ban on lead } \\
\text { shot in wet- } \\
\text { lands or for } \\
\text { waterfowl }\end{array}$ & $\begin{array}{l}\text { Ban on lead } \\
\text { shot for all } \\
\text { hunting }\end{array}$ & $\begin{array}{l}\text { Partial ban } \\
\text { on lead } \\
\text { ammunition }\end{array}$ & $\begin{array}{l}\text { Ban on all } \\
\text { forms of lead } \\
\text { ammunition }\end{array}$ & $\begin{array}{l}\text { Ban on } \\
\text { hunting }\end{array}$ & $\begin{array}{l}\text { Nontoxic } \\
\text { shot regu- } \\
\text { lations in } \\
\text { addition to } \\
\text { Federal }\end{array}$ \\
\hline *Alaska & & & & & & & & Yes \\
\hline \multicolumn{9}{|l|}{${ }^{*}$ Arizona } \\
\hline \multicolumn{9}{|l|}{${ }^{\star}$ Arkansas } \\
\hline${ }^{\star}$ California & & & & & $\begin{array}{l}\text { Banned in } \\
\text { Condor range } \\
2008\end{array}$ & & & Yes \\
\hline \multicolumn{9}{|l|}{${ }^{*}$ Colorado } \\
\hline \multicolumn{9}{|l|}{${ }^{\star}$ Connecticut } \\
\hline \multicolumn{9}{|l|}{ *Delaware } \\
\hline \multicolumn{9}{|l|}{${ }^{*}$ Florida } \\
\hline \multicolumn{9}{|l|}{${ }^{*}$ Georgia } \\
\hline \multicolumn{9}{|l|}{ *Hawaii } \\
\hline \multicolumn{9}{|l|}{ *Idaho } \\
\hline *Illinois & & & & & & & & Yes \\
\hline \multicolumn{9}{|l|}{ *Indiana } \\
\hline *lowa & & & & & & & & Yes \\
\hline *Kansas & & & & & & & & Yes \\
\hline *Kentucky & & & & & & & & Yes \\
\hline *Louisiana & & & & & & & & Yes \\
\hline *Maine & & & & & & & & Yes \\
\hline *Maryland & & & & & & & & Yes \\
\hline${ }^{*}$ Massachusetts & & & & & & & & Yes \\
\hline *Michigan & & & & & & & & Yes \\
\hline${ }^{*}$ Minnesota & & & & & & & & Yes \\
\hline \multicolumn{9}{|l|}{ *Mississippi } \\
\hline${ }^{*}$ Missouri & & & & & & & & Yes \\
\hline \multicolumn{9}{|l|}{ *Montana } \\
\hline *Nebraska & & & & & & & & Yes \\
\hline \multicolumn{9}{|l|}{${ }^{*}$ Nevada } \\
\hline${ }^{*}$ New Hampshire & & & & & & & & \\
\hline
\end{tabular}


- LEAD AMMUNITION REGULATION -

\begin{tabular}{|c|c|c|c|c|c|c|c|c|}
\hline $\begin{array}{l}\text { Country } \\
\text { or State }\end{array}$ & $\begin{array}{l}\text { Recommended use } \\
\text { of nontoxic shot }\end{array}$ & $\begin{array}{l}\text { Partial ban } \\
\text { on lead shot }\end{array}$ & $\begin{array}{l}\text { Ban on lead } \\
\text { shot in wet- } \\
\text { lands or for } \\
\text { waterfowl }\end{array}$ & $\begin{array}{l}\text { Ban on lead } \\
\text { shot for all } \\
\text { hunting }\end{array}$ & $\begin{array}{l}\text { Partial ban } \\
\text { on lead } \\
\text { ammunition }\end{array}$ & $\begin{array}{l}\text { Ban on all } \\
\text { forms of lead } \\
\text { ammunition }\end{array}$ & $\begin{array}{l}\text { Ban on } \\
\text { hunting }\end{array}$ & $\begin{array}{l}\text { Nontoxic } \\
\text { shot regu- } \\
\text { lations in } \\
\text { addition to } \\
\text { Federal }\end{array}$ \\
\hline${ }^{*}$ New Jersey & & & & & & & & Yes \\
\hline${ }^{*}$ New Mexico & & & & & & & & Yes \\
\hline${ }^{\star}$ New York & & & & & & & & Yes \\
\hline${ }^{*}$ North Carolina & & & & & & & & Yes \\
\hline *North Dakota & & & & & & & & Yes \\
\hline${ }^{*}$ Ohio & & & & & & & & Yes \\
\hline \multicolumn{9}{|l|}{ *Oklahoma } \\
\hline *Oregon & & & & & & & & Yes \\
\hline \multicolumn{9}{|l|}{ *Pennsylvania } \\
\hline \multicolumn{9}{|l|}{${ }^{*}$ Rhode Island } \\
\hline \multicolumn{9}{|l|}{ *South Carolina } \\
\hline *South Dakota & & & & & & & & Yes \\
\hline \multicolumn{9}{|l|}{ *Tennessee } \\
\hline \multicolumn{9}{|l|}{ *Texas } \\
\hline *Utah & & & & & & & & Yes \\
\hline \multicolumn{9}{|l|}{ *Vermont } \\
\hline \multicolumn{9}{|l|}{ *Virginia } \\
\hline *Washington & & & & & & & & Yes \\
\hline *Wyoming & & & & & & & & Yes \\
\hline
\end{tabular}




\section{- AVERY AND WATSON -}

Table 2. Regulation of lead ammunition over time.

\begin{tabular}{|c|c|}
\hline Date & Country and type of regulation \\
\hline 1975 & Mauritania hunting laws prohibit use of toxic ammunition for large game and sport hunting. \\
\hline 1980 & Liberia bans lead shot due to military coup. \\
\hline 1985 & Denmark hunters initiate use of nontoxic shot. \\
\hline \multicolumn{2}{|r|}{ 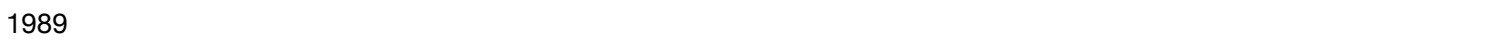 } \\
\hline \multicolumn{2}{|l|}{1990} \\
\hline \multirow[t]{2}{*}{1991} & USA bans the use of lead shot over wetlands. \\
\hline & Norway bans lead shot in wetlands for hunting of all ducks, geese, and waders. \\
\hline \multicolumn{2}{|l|}{1992} \\
\hline \multirow[t]{7}{*}{1993} & South Australia, Australia bans the use of lead shot. \\
\hline & Victoria, Australia bans the use of lead shot during duck season. \\
\hline & Denmark bans the use of lead shot over wetlands. \\
\hline & Cyprus bans the use of lead shot over wetlands. \\
\hline & $\begin{array}{l}\text { Germany bans the use of lead shot over wetlands in } 8 \text { Lander and recommends voluntary use of nontoxic shot } \\
\text { over all wetlands. }\end{array}$ \\
\hline & Belgium bans the use of lead shot over Ramsar wetlands. \\
\hline & February-Netherlands bans the use of lead shot for hunting over wetlands. \\
\hline \multicolumn{2}{|l|}{1994} \\
\hline \multirow[t]{3}{*}{1995} & Victoria, Australia bans the use of lead shot for duck hunting. \\
\hline & Netherlands bans the use of lead shot in all hunting. \\
\hline & UK instills voluntary use of nontoxic shot over wetlands. \\
\hline \multirow[t]{2}{*}{1996} & Denmark bans the use of lead shot in all hunting. \\
\hline & Finland bans the use of lead shot over wetlands. \\
\hline 1997 & Canada bans the use of lead shot for hunting migratory game birds near water. \\
\hline \multirow[t]{3}{*}{1998} & Switzerland bans the use of lead shot for hunting over wetlands and shallow water areas. \\
\hline & Belgium bans the use of lead shot over all wetlands. \\
\hline & Northern Territory, Australia bans the use of lead shot during duck season. \\
\hline \multirow[t]{2}{*}{1999} & England prohibits use of lead shot over wetlands and for all waterfowl. \\
\hline & Canada bans the use of lead shot for hunting all migratory game birds (with a few exceptions). \\
\hline \multirow[t]{4}{*}{2000} & Japan bans the use of lead bullets for deer hunting in Hokkaido. \\
\hline & Latvia bans the use of lead shot over wetland special protected areas. \\
\hline & Spain bans the use of lead shot at Ramsar sites. \\
\hline & Denmark bans the import of all lead products including ammunition. \\
\hline 2001 & Queensland, Australia instills voluntary ban on the use of lead shot over wetlands. \\
\hline \multirow[t]{3}{*}{2002} & Sweden bans the use of lead shot over wetlands. \\
\hline & Wales bans the use of lead shot over wetland sites of special scientific interest. \\
\hline & Sweden bans the use of lead shot for clay pigeons. \\
\hline \multicolumn{2}{|l|}{2003} \\
\hline \multirow[t]{2}{*}{2004} & Netherlands bans the use of lead shot for clay pigeons. \\
\hline & Tasmania, Australia bans the use of lead shot over public wetlands and Crown Land. \\
\hline \multirow[t]{2}{*}{2005} & Hungary bans the use of lead shot over wetlands. \\
\hline & 31 March-Scotland bans the use of lead shot over wetlands. \\
\hline \multirow{2}{*}{2006} & Norway bans the use of lead shot for all hunting. \\
\hline & New Zealand bans 10 and 12 gauge shot for waterfowl near water. \\
\hline \multirow{3}{*}{2007} & \\
\hline & Camp Roberts, California, USA bans all lead ammunition for hunting. \\
\hline & Fort Hunter Liggett, California, USA bans lead ammunition for hunting. \\
\hline \multirow[t]{6}{*}{2008} & Tejon Ranch, California, USA bans all lead ammunition for hunting. \\
\hline & Camp Roberts, California, USA bans use of all lead shot and ammunition for hunting. \\
\hline & $\begin{array}{l}\text { California, USA bans the use of lead ammunition when taking big game and coyotes in the California Condor } \\
\text { range in California. }\end{array}$ \\
\hline & Sweden enacts a total ban on lead shot and ammunition. \\
\hline & Belgium considers a total ban on the use of lead shot. \\
\hline & Portugal proposes a ban on the use of lead shot in wetlands. \\
\hline
\end{tabular}

